\title{
AQUISIÇÃO DE VOCABULÁRIO: EFEITO DE ESTÍMULOS NOVOS NO CONTROLE DE RESPOSTAS
}

\author{
VOCABULARY ACQUISITION: EFFECT OF NEW STIMULI ON \\ CONTROL OF RESPONSES
}

\author{
Marcileyde Tizo \& Lorismário E. Simonassi \\ UNIVERSIDADE CATÓLICA DE GOIÁS, BRASIL
}

\begin{abstract}
RESUMO
O presente estudo verificou em três experimentos como crianças em seus primeiros anos de vida aprendem a relacionar palavras novas a objetos ou eventos. O Experimento 1, com 10 crianças de 2,5 a 4 anos, verificou os efeitos sobre o responder: a) da presença de uma figura nova quando uma palavra nova era ditada; e b) do acréscimo da terminaçáo de plural em uma palavra nova ditada na presença de um conjunto de figuras de um mesmo estímulo novo. Os resultados mostraram que o estímulo novo controlou o responder da maioria das crianças, independente da dica lexical. O Experimento 2, realizado com seis crianças de 2 anos e dois meses a 3 anos e seis meses de idade, avaliou se a substituição dos estímulos conhecidos por outros estímulos conhecidos reduziria a utilização desses estímulos nas respostas aos novos estímulos. Observou-se, como no Experimento 1 , que as crianças continuaram respondendo nas figuras conhecidas quando uma palavra nova era ditada. $\mathrm{O}$ Experimento 3 verificou se a alteração da posição dos estímulos de comparação modificaria o responder de quatro crianças com 2 anos e oito meses a 4 anos de idade. Observou-se uma diminuição significativa de respostas para as figuras conhecidas quando uma palavra nova era ditada. Concluiu-se que a presença do estímulo "novo" controlou o comportamento das crianças durante os três experimentos realizados e que a dica lexical não exerceu controle sobre a resposta.
\end{abstract}

Palavras-chave: comportamento verbal falado, novidade, pareamento com o modelo.

\begin{abstract}
The present study investigated in three experiments how children in their first years of life learn to relate new words to objects or events. The first experiment, with ten children aged between 2.5 and 4 years, investigated the behavioral effects of: a) a new picture present when a new word was dictated; and b) inclusion of the plural ending in a new word (lexical tip) when it was spoken in the presence of a set of figures of the same stimulus. Results showed that new stimuli controlled the majority of responses despite the lexical tip. Some responses of known stimuli to new spoken words were observed in the first and second experiments. Experiment 2, conducted with six children (ages from 2.2 to 3.6 years), investigated the effects of replacing known stimuli with other known pictures on responding to new spoken words. Results were similar to the first experiment: children continued to respond with known pictures to new spoken words. The third experiment was intended to investigate whether responses were modified by repositioning the comparative stimuli of four children aged from 2.7 to 4 years old. A significant reduction of responding with known pictures to new spoken words was observed. In conclusion, new stimuli controlled children's behavior during all three experiments, and lexical tips did not control the observed responses.
\end{abstract}

Key words: spoken verbal behavior, novelty, pairing with model.

Nos últimos anos, têm sido realizados vários estudos por profissionais de diferentes áreas e abordagens teóricas quanto à aquisição de vocabulário (Vincent-Smith, Bricker

\footnotetext{
Agradecemos ao Prof. Dr. Weber Martins pela realização do Software; ao Raphael Cardoso pela ajuda na realização do teste estatístico; a instituição que abriu as portas para a realização da pesquisa. Agradeço aos Profs. Drs. João Claudio Todorov, Aline R. A. da Costa, Ilma A. G. S. Britto e Ângela M. M. Duarte pelas contribuições apresentadas na defesa dessa dissertação de mestrado. Endereço para correspondência: Lorismário Simonassi, Rua Fortaleza, ${ }^{\circ}$. 355 Apt. 104 Ed. Fernanda. Bairro/Setor: Alto da Glória. CEP.: 74810-120, Goiânia, GO. Telefone: (xxx-62) 3246-4817. E-mail: lorismario@ucg.br.
} 
\& Bricker, 1974; Dixon, 1977; Carey, 1982; de Rose, de Souza, Rossito \& de Rose, 1992; Golinkoff, Mervis \& Hirsh-Pasek, 1994; Mervis \& Bertrand, 1995; Wilkinson, Dube \& McIlvane, 1996; Wilkinson \& McIlvane, 1997; McIlvane, Wilkinson \& de Souza, 2000; Costa, Wilkinson, McIlvane \& de Souza, 2001; Costa e de Souza, 2003). Entre esses estudos surgiu uma nova perspectiva sobre a aprendizagem da fala quanto à nomeação de objetos e eventos. Esses estudos sobre a aquisição de vocabulário investigam como ocorre a aquisição de palavras novas, utilizando metodologias que empregam a introdução dessas palavras novas. Tais palavras são "sem sentido" e criadas pelo examinador.

O que tem sido observado, nos diferentes estudos, é que em situação experimental, na qual as crianças são colocadas frente a objetos conhecidos e um objeto novo, há sistematicamente a escolha do objeto novo quando as crianças ouvem um nome também novo, (Wilkinson \& McIlvane, 1997).

Apesar deste resultado obtido experimentalmente (a escolha de uma figura ou objeto indefinido quando um nome indefinido é ditado) ter sido observado com diferentes populações de crianças, quanto a idade, nacionalidade, nível de desenvolvimento intelectual e escolaridade (de Rose, de Souza, Rossito \& de Rose, 1992; de Rose, de Souza \& Hanna, 1996; Dixon, 1977; Golinkoff \& cols., 1994; Hutchinson, 1986; Mervis \& Bertrand, 1994; McIlvane, Bass, O’ Brien, Gerovac, \& Stoddard, 1984; McIlvane, Kledaras, Lowry, \& Stoddard, 1992; McIlvane, Kledaras, Munson, King, de Rose \& Stoddard, 1987; McIlvane, Munson \& Stoddard, 1988; McIlvane \& Stoddard, 1981; Stromer, 1989; Wilkinson \& McIlvane, 1997; Wilkinson, Dube \& McIlvane, 1998), surgiu uma dúvida quanto a generalidade desse tipo de aprendizagem já que o mesmo não é adaptativo em algumas situações de aquisição de vocabulário. A eventual fixação em uma única correspondência nome-referente levaria o indivíduo a rejeitar mais de um nome para um único referente e atrapalharia a aquisição de palavras como sinônimo, adjetivo e nomes para partes de um objeto, animal ou evento (Costa, de Rose \& de Souza, submetido).

Assim, Costa e de Souza (2003) levantaram a hipótese de que além de atribuir nomes novos a objetos ou eventos também novos, a aprendizagem de vocabulário por crianças pode ser influenciada pelas características constantes, presentes no discurso oral da comunidade verbal, que se repetem diferencialmente na presença dos diferentes tipos de classes lexicais (verbo, adjetivo, substantivo, plurais, etc.) ao longo da história de aquisição de vocabulário. De acordo com essa perspectiva, no início do desenvolvimento verbal o comportamento da criança pode ser influenciado por dicas caso estas estejam presentes no contexto em que a palavra nova é ditada. O presente experimento foi realizado com o objetivo de verificar essa hipótese.

\section{EXPERIMENTO I}

Os objetivos do Experimento 1 foram verificar se: a) a presença da figura nova quando uma palavra nova é ditada controla o responder; b) o acréscimo da terminação de plural em uma palavra nova controla o responder quando ditada na presença de várias figuras de um mesmo estímulo novo agrupadas indicando um único estímulo de comparação; e c) aumentando-se as oportunidades de escolha o responder se modifica.

\section{MÉTOdo}

\section{Participantes}

Participaram do estudo 10 crianças (2 anos e meio a 4 anos de idade), todas sem relato dos responsáveis quanto a problemas 
relacionados ao desenvolvimento da linguagem, e atividades motoras, auditivas, visuais e sem qualquer influência de dicas lexicais em seu repertório.

Foi realizado o contato com as crianças por meio da própria instituição em que elas se encontravam. A duração da participação e o número de sessões dependeram do ritmo de cada participante, mas a duração média foi de 20 minutos.

A participação no presente estudo ocorreu mediante consentimento prévio dos responsáveis, atestado pela assinatura do Termo de Consentimento Livre e Esclarecido, submetido e aprovado pelo Comitê de Ética e Pesquisa da instituição.

\section{Situação e materiais}

As sessões experimentais foram realizadas em uma instituição (berçário) na cidade de Goiânia, Go. Utilizou-se, para a programação das atividades, um microcomputador com tela sensível ao toque, mesa e cadeira proporcionais ao tamanho da criança. Foi utilizado o software "Aquisição de Vocabulário Infantil versão 1.0” (Martins, 2008), implementado em linguagem Visual Basic for Applications na planilha eletrônica Microsoft Excel.

Os estímulos experimentais eram visuais (figuras do pato, cachorro e coelho, figuras novas ou sem sentido e um quadrado preto) e auditivos. Essas figuras eram apresentadas na tela do computador dentro de três a quatro quadrados de $5 \mathrm{~cm}$, dispostos no centro da tela, do lado direito, esquerdo, superior ou inferior. A combinação das figuras apresentadas na tela do computador alterava-se de tentativa a tentativa. As palavras foram gravadas e ditadas através de um autofalante. O fundo da tela era amarelo claro e as figuras eram em preto e branco.

\section{Procedimento}

As tarefas básicas consistiram em tentativas de discriminação condicional em que o estímulo modelo era uma palavra ditada e os estímulos de comparação eram figuras disponíveis no centro da tela do computador.

A criança respondia tocando na tela do computador sobre uma das figuras - estímulo de comparação - disponível em cada tentativa após o modelo auditivo ter sido apresentado. Foram apresentadas consequências diferenciais para as respostas corretas e incorretas ao longo das tentativas, isto é, as respostas corretas foram seguidas pela apresentação de vários quadrados coloridos que mudavam de core e ficavam piscando na tela. A consequência para as respostas incorretas foi o escurecimento da tela por 2 segundos.

As etapas realizadas nesse estudo foram: análise de repertório, pré-treino, treino e teste, como descritas abaixo.

Análise do repertório. Foi realizada em duas fases. Na primeira, verificou-se se existia ou não a influência da dica lexical "s" no repertório da criança. Apresentaram-se, primeiramente, três cartões $(10 \times 10 \mathrm{~cm})$ com figuras conhecidas. No primeiro cartão, foi apresentada a figura do cachorro, no segundo a figura do pato e no terceiro a figura do coelho. Em outros três cartões, foram apresentadas as figuras de patos, de coelhos e de cachorros. Por exemplo, três figuras de cachorro foram desenhadas em um papel cartão de $10 \mathrm{~cm}$. Cada cartão foi apresentado individualmente e seguido pela instrução verbal: "O que é isso?" ou "Que figura é essa?”. Nenhuma dica vocal (ex: é um animal) foi apresentada pelo experimentador.

$\mathrm{Na}$ segunda fase, para verificar se as figuras denominadas pelo experimentador como novas (isto é, não familiares, com formas distintas às figuras conhecidas) eram de fato novas para a criança. Foram apresentados 
cartões com essas figuras novas. A apresentação do cartão era seguida pela instrução "O que é isso?". Participaram das etapas de treino e teste as crianças que não apresentaram influência da dica lexical "s" em seu repertório, isto é, não nomearam as figuras no plural (ex: "cachorros", "titios", etc).

Pré-treino para o responder. O pré-treino constou de seis tentativas. Cada tentativa tinha um estímulo de comparação no centro da tela. Nas três primeiras tentativas, apresentou-se o estímulo de comparação no centro da tela, seguido pela instrução verbal: "Olhe a figura na tela. Agora, coloque o dedo em cima do cachorro". O toque sobre a figura fazia com que a consequência para a resposta correta fosse apresentada, isto é, quadrados coloridos piscando no centro da tela e consequência social como: "Muito bem!" ou "Isso mesmo!"

Após essas três tentativas, o participante realizou mais três tentativas com as mesmas configurações das três tentativas anteriores, sendo que o som era emitido a cada dois segundos como: "cachorro", "coelho" ou "pato".

Ao realizar as seis tentativas adequadamente, o participante iniciou as etapas de treino. Caso necessário, o participante realizava novamente todo o procedimento de pré-treino.

\section{Treino para o responder.}

Etapa 1. Estabelecimento das discriminações condicionais: emparelhamento auditivo visual.

Nessa etapa, foi ensinada a tarefa de tocar na tela sobre uma das três figuras (cachorro, pato e coelho) após o modelo ter sido ditado. Foram realizadas nove tentativas. As figuras alternavam de posição na tela, a cada tentativa.

Estabeleceu-se um critério de 100\% de acertos para passar para a próxima etapa; enquanto o critério não foi atingido, a criança continuou na etapa. Portanto, o número de blocos a serem realizados nesta etapa dependia do desempenho da criança, com a ressalva de que não mais de três blocos eram realizados na mesma etapa. Nessa etapa as respostas eram consequenciadas continuamente (CRF).

Etapa 2. Introdução gradual de uma máscara no lugar de um dos estímulos de comparação.

Nesta etapa, um pequeno quadrado preto passou a ser sobreposto sobre uma das figuras de comparação. O tamanho do quadrado foi aumentando ao longo das 12 tentativas de aproximações sucessivas de introdução gradual (fading in) de uma máscara, de modo que na tentativa final o quadrado preto cobriu totalmente uma das figuras, ficando disponíveis para a escolha, portanto, duas figuras e um quadrado preto. O critério e as consequências foram semelhantes à Etapa 1.

Etapa 3. Máscara como um estímulo de comparação

Nesta etapa a máscara completa apareceu desde a primeira tentativa como uma das alternativas de escolha. Esta sessão era composta por 12 tentativas. O critério e as consequências foram como na Etapa 1.

Etapa 4. Introdução da consequenciação em esquema intermitente (VR2).

Nesta etapa, a máscara apareceu desde o início como na Etapa 3, mas as consequências para acertos foram apresentadas em VR2, isto é, os quadrados só apareceram em média a cada duas respostas corretas. A etapa tinha 12 tentativas e foi requerido um critério de 100\% de respostas corretas na etapa, para considerar instalada uma linha de base para o responder.

\section{Testes}

Etapa 1. Teste do responder na presença de palavra nova e figura nova.

Nessa etapa foi realizado um bloco de treino em VR2 (idêntico ao Treino 4) com o acréscimo de 
tentativas de teste intercaladas ao mesmo. Em cada tentativa de teste foram apresentados três estímulos de comparação na tela compostos por: figura nova, figura conhecida e máscara, assim como, figura conhecida, figura conhecida e máscara.

Nenhuma consequência programada seguiu o responder nas tentativas de teste e as tentativas de treino continuaram sendo consequenciadas em VR2. Isso para todas as Etapas de teste (1, 2 e 3).

Etapa 2. Teste do responder na presença de palavra nova com a terminação "s" e figura nova e/ou figura com três exemplos novos.

As quatro tentativas de teste foram distribuídas entre 10 tentativas de treino. Em cada tentativa de teste foram apresentados três estímulos de comparação na tela compostos por: figura com três exemplos novos, figura nova e máscara, assim como, figura conhecida, figura nova e máscara.

Etapa 3. Teste do responder na presença de palavra nova com terminação "s" e figura nova e/ou figura com três exemplos novos. As tentativas foram compostas por quatro estímulos de comparação.

Nessa Etapa 3 do teste distribuiu-se entre as tentativas de treino em VR2 as seis tentativas de teste. Cada tentativa de teste tinha quatro estímulos de comparação compostos por: figura com três exemplos novos, figura nova, figura conhecida e máscara, assim como, figura nova, figura conhecida, figura conhecida e máscara.

\section{RESUlTADOS}

A Tabela 1 mostra as respostas emitidas pelos participantes quando uma palavra nova foi ditada na presença de três estímulos de comparação durante a Etapa 1 do teste. Os resultados das tentativas 1 e 3 , assim como das tentativas 2 e 4 foram apresentadas e analisadas juntos, pois os estímulos que as compunham eram: novos, conhecidos ou máscara, como se pode observar na Tabela 1.
Tabela 1

Porcentagem de respostas corretas apresentadas na Etapa 1 do Experimento 1

\begin{tabular}{|c|c|c|c|c|c|c|}
\hline \multirow{2}{*}{$\begin{array}{l}\text { Estímulos de } \\
\text { comparação }\end{array}$} & \multicolumn{3}{|c|}{ Tentativas 1 e 3} & \multicolumn{3}{|c|}{ Tentativas 2 e 4} \\
\hline & M & $\mathrm{N}$ & C & M & C1 & C2 \\
\hline$\overline{R e s u l t a d o s}$ & 4 & 11 & 5 & 12 & 5 & 3 \\
\hline$\%$ & 20,0 & 55,0 & 25,0 & 60,0 & 25,0 & 15,0 \\
\hline
\end{tabular}

Observa-se que nas tentativas 1 e 3 compostas por máscara $(\mathrm{M})$, figura nova $(\mathrm{N})$ e figura pato/cachorro (C), obteve-se 11 repostas para a figura nova (55\%), cinco respostas para a figura pato/cachorro (25\%) e quatro respostas para a máscara $(20 \%)$. Nas tentativas 2 e 4 compostas por máscara e duas figuras conhecidas (pato-C1/coelho-C2), obteve-se 12 respostas para a máscara $(60 \%)$, cinco respostas para a figura do pato $(25 \%)$ e três respostas para a figura do coelho (15\%).

A Tabela 2 apresenta as respostas emitidas pelos participantes quando uma palavra nova acompanhada de "s" foi ditada na presença de três estímulos de comparação. Nas tentativas 1 e 3 os participantes emitiram oito respostas para a figura nova (40\%), 12 respostas para a figura com três exemplos novos ou $\mathrm{N}+\mathrm{s}(60 \%)$ e para a máscara não ocorreram respostas. Nas tentativas 2 e 4 , foram emitidas 18 respostas para a figura nova $(90 \%)$, duas respostas para a máscara $(10 \%)$ e nenhuma resposta foi emitida para a figura conhecida.

Como mostra a Tabela 3, quando uma palavra nova acompanhada de "s" foi ditada na presença de quatro estímulos de comparação, obtiveram-se nas tentativas 1, 3, 4 e 6, 18 respostas para a figura nova (45\%), 16 respostas para a figura com três exemplos novos (40\%), cinco respostas para a figura conhecida do pato/coelho (12,5\%) e uma resposta para a máscara (2,5\%). E nas tentativas 2 e 5 , obtiveram 18 respostas para a figura nova (90\%), uma 
resposta para a máscara $(5 \%)$ e uma resposta para a figura do pato $(5 \%)$.

\section{Tabela 2}

Porcentagem de respostas corretas apresentadas na Etapa 2 do Experimento 1

\begin{tabular}{|c|c|c|c|c|c|c|}
\hline \multirow{2}{*}{$\begin{array}{l}\text { Estímulos de } \\
\text { comparação }\end{array}$} & \multicolumn{3}{|c|}{ Tentativas 1 e 3} & \multicolumn{3}{|c|}{ Tentativas 2 e 4} \\
\hline & M & $\mathrm{N}$ & $\mathrm{N}+\mathrm{s}$ & M & $\mathrm{N}$ & $\mathrm{C}$ \\
\hline Resultados & 0 & 8 & 12 & 2 & 18 & 0 \\
\hline$\%$ & 0 & 40,0 & 60,0 & 10,0 & 90,0 & 0 \\
\hline
\end{tabular}

Tabela 3

Porcentagem de respostas corretas apresentadas na Etapa 3 do Experimento 1.

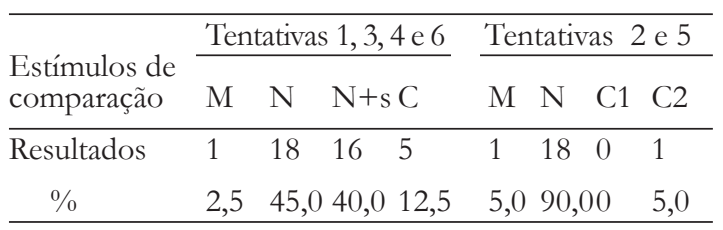

Observa-se que entre as respostas emitidas pelos participantes durante as Etapas 1 e 3 do teste (Tabelas 1 e 3) obteve uma alta frequência de respostas para as figuras conhecidas quando um estímulo novo foi ditado. A soma das respostas para figuras conhecidas durante as Etapas 1 e 3 foi de 19 respostas.

\section{Discussão}

Os resultados mostram que o estímulo novo controlou o comportamento dos participantes no teste. Os dados obtidos nas Etapas 2 e 3 do teste, mostram que o acréscimo da terminação " $\mathrm{s"} \mathrm{na}$ palavra nova não controlou o responder quando ditada na presença de várias figuras de um mesmo estímulo novo agrupadas indicando um único estímulo de comparação. Quando uma palavra nova com terminação "s" (ex: "bassaxos") foi ditada, geralmente as respostas dos participantes ficaram distribuídas entre a figura nova e figura com três exemplos novos. E quando nas tentativas 2 e 4 a palavra nova com terminação "s" foi ditada, obteve-se alta frequência de respostas para a figura nova. Esses dados mostram que o estímulo novo controlou o responder, mas o mesmo não se pode dizer quanto à terminação "s" no final da palavra, pois os participantes ainda não aprenderam a responder ao léxico "s" e por isso podem estar respondendo a esse estímulo por ser "novo" e não devido a presença da dica lexical. Supõe-se que o que pode ter controlado o responder é a propriedade que essas figuras compartilham, o fato de serem "novas" (sem sentido, inventadas pelo experimentador), independente da dica lexical.

Um dado obtido nesse estudo e que se difere dos estudos anteriores (Wilkinson \& McIlvane, 1997; Costa \& de Souza, 2003) foram as respostas que os participantes emitiram para figuras conhecidas quando uma palavra nova foi ditada. Esses estudos anteriores mostraram que todos os participantes selecionavam um estímulo novo quando uma palavra nova foi ditada. Já no presente estudo, a escolha para estímulos conhecidos ocorreram por duas ou três vezes durante as Etapas 1 e 3 do teste. O que pode ter controlado o comportamento desses participantes quando responderam para figuras conhecidas quando palavras novas foram ditadas?

Uma possibilidade de explicação para essa questão é que o comportamento de selecionar a figura conhecida quando uma palavra nova foi ditada durante as etapas de teste pode estar relacionado ao desempenho obtido no treino, em que o critério de $100 \%$ de acertos não foi atingido por três participantes ( $\operatorname{Pp~3,~} 5$ e 6).

Outra possibilidade é que na Linha de Base (Análise do repertório), no início do estudo, dois dos participantes (não identificados 
dentre os participantes pelo experimentador) não nomearam a figura do coelho e do pato. Assim, supõe-se que essas figuras "pato" e "coelho" apresentadas durante o teste poderiam ser "desconhecidas", uma vez que, as respostas emitidas pelos participantes durante as etapas de teste foram apresentadas para as figuras do pato e do coelho .

\section{EXPERIMENTO 2}

Esse experimento verificou se substituindo os estímulos conhecidos (coelho e pato) por outras figuras conhecidas (bola e casa) e atingindo o critério de $100 \%$ de acertos no treino, poderia ocorrer a diminuição na frequência de respostas para figuras conhecidas quando uma palavra nova fosse ditada.

\section{MÉTodo}

\section{Participantes}

Foi realizado com outras seis crianças, com idades entre 2 anos e dois meses a 3 anos e seis meses.

\section{Situação e materiais}

Para a realização do Experimento 2 substituiu-se as figuras do pato e do coelho (do Experimento 1) pelas figuras da bola e da casa. As demais ocorreram como no Experimento 1.

\section{Procedimento}

O Experimento 2 foi semelhante ao Experimento 1, sendo que na Fase 1 da Análise de Repertório foram apresentados os cartões com figuras conhecidas (cachorro, bola e casa) com a instrução verbal: "O que é isso?", as crianças deveriam emitir respostas relacionadas a figura conhecida apresentada no cartão, mas caso isso não ocorresse era realizada com a criança a Etapa 1 do pré-treino como descrito no Experimento 1. Após esse pré-treino o experimentador mostrou novamente os cartões com as figuras conhecidas à criança. Continuaram no estudo, as crianças que nomearam as figuras apresentadas. Aquelas que não o fizeram foram excluídas.

As etapas de treino e teste foram semelhantes ao Experimento 1.

\section{Resultados}

A estrutura das tabelas apresentadas no Experimento 2 foi semelhante a do Experimento 1.

\section{Tabela 4}

Porcentagem de respostas corretas apresentadas na Etapa 1 do Experimento 2

\begin{tabular}{llllllll}
\hline \multirow{2}{*}{$\begin{array}{l}\text { Estímulos de } \\
\text { comparação }\end{array}$} & \multicolumn{3}{l}{ M } & Nentativas 1 e 3 & & \multicolumn{3}{c}{ Tentativas 2 e 4 } & & M & C1 & C2 \\
\hline Resultados & 2 & 7 & 3 & & 10 & 0 & 2 \\
$\%$ & 16,6 & 58,3 & 25,0 & & 83,3 & 0 & 16,6 \\
\hline
\end{tabular}

A Tabela 4 mostra que quando uma palavra nova foi ditada na presença de três estímulos de comparação, obteve-se sete respostas para a figura nova $(58,3 \%)$, três respostas para a figura do cachorro/bola (25\%) e duas respostas para a máscara $(16,6 \%)$ nas tentativas 1 e 3 . Nas tentativas 2 e 4 , obteve-se 10 respostas para a máscara $(83,3 \%)$ e duas respostas para a figura da bola $(16,6 \%)$.

$\mathrm{Na}$ Tabela 5, abaixo, quando uma palavra nova acompanhada de "s" foi ditada obteve-se nas tentativas 1 e 3 , seis respostas para a figura com três exemplos novos (50\%), cinco respostas para a figura nova $(41,6 \%)$ e uma resposta para a máscara $(8,3 \%)$. E nas tentativas 2 e 4 , obteve 10 respostas para a figura nova $(83,3 \%)$, uma resposta pra a máscara $(8,3 \%)$ e uma resposta para a figura conhecida $(8,3 \%)$. 


\section{TABEla 5}

Porcentagem de respostas corretas apresentadas na Etapa 2 do Experimento 2

\begin{tabular}{|c|c|c|c|c|c|c|}
\hline \multirow{2}{*}{$\begin{array}{l}\text { Estímulos de } \\
\text { comparação }\end{array}$} & \multicolumn{3}{|c|}{ Tentativas 1 e 3} & \multicolumn{3}{|c|}{ Tentativas 2 e 4} \\
\hline & M & $\mathrm{N}$ & $\mathrm{N}+\mathrm{s}$ & M & $\mathrm{N}$ & $\mathrm{C}$ \\
\hline Resultados & 1 & 5 & 6 & 1 & 10 & 1 \\
\hline$\%$ & 8,3 & 41,6 & 50,0 & 8,3 & 83,3 & 8,3 \\
\hline
\end{tabular}

Na Tabela 6 observa-se que quando uma palavra nova acompanhada de "s" foi ditada na presença de quatro estímulos de comparação, obteve-se nas tentativas 1 e 3, 17 respostas para a figura com três exemplos novos (70,8\%), seis respostas para a figura nova $(25 \%)$ e uma resposta para a figura do cachorro (4,16\%). Nas tentativas 2 e 5, obteve-se nove respostas para a figura nova $(75 \%)$, duas respostas para a figura da casa $(16,6 \%)$ e uma resposta para a figura bola $(8,3 \%)$. Nenhuma resposta foi obtida para a máscara dentre todas essas tentativas da Etapa 3 de teste.

Tabela 6

Porcentagem de respostas corretas apresentadas na Etapa 3 do Experimento 2

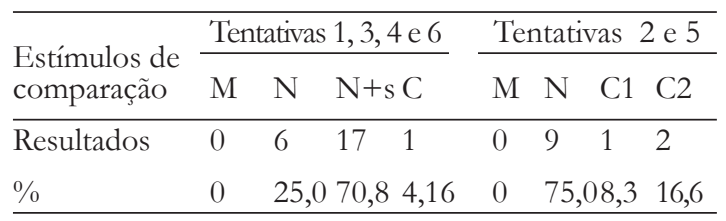

Observando as Tabelas 4, 5 e 6, obtêm um total de 12 respostas para figuras conhecidas durante as Etapas 1, 2 e 3 do teste no Experimento 2 , sendo duas respostas para a figura do cachorro, duas respostas para a figura da casa e oito respostas para a figura da bola. Todos os três estímulos conhecidos apresentados foram selecionados quando uma palavra nova foi ditada nas etapas de teste.

\section{Discussão}

Os resultados mostraram que a hipótese levantada de que as figuras conhecidas apresentadas no Experimento 1 poderiam ser desconhecidas ao participante é falsa, pois os participantes continuaram respondendo para as figuras conhecidas quando uma palavra nova foi ditada no Experimento 2.

Uma questão observada no Experimento 2 e que deve ser verificada foi: quando um estímulo modelo foi ditado, o participante respondeu tocando sobre a figura correspondente ao som emitido, mas ainda no treino quando o participante tocava uma vez sobre a figura e por não tocar adequadamente (isto é, não registrar uma resposta e apresentar uma nova tentativa) o experimentador pedia ao participante para tocar novamente sobre a figura para que a consequência para o responder fosse apresentada. Com esse "tocar novamente", o participante começou a tocar uma vez, duas ou três vezes sobre uma figura quando um som era apresentado. E esse comportamento de tocar várias vezes sobre uma figura resultou em novas respostas a tentativas seguintes, isto é, geralmente esse segundo ou terceiro toque sobre a figura resultava em novas respostas, sendo registradas automaticamente como correspondentes a tentativas seguintes, portanto um erro induzido pela instrução ("tocar novamente") do experimentador e pela configuração do equipamento. Os estímulos de comparação, a cada tentativa, encontravam-se na mesma posição.

Diante da hipótese de que comportamento aprendido (dois a três toques sobre um estímulo de comparação) durante o experimento pode ter comprometido a resposta do participante na tentativa seguinte, 
isso pode ser devido à posição em que estes estímulos de comparação foram arranjados. O que ocorreria nos testes se a posição dos estímulos de comparação a cada tentativa fosse diferente?

\section{EXPERIMENTO 3}

Esse estudo verificou se, alterando a posição dos estímulos de comparação a cada tentativa, o responder se modificaria.

\section{MÉTodo}

\section{Participante}

Foi realizado com outras quatro crianças com 2 anos e oito meses a 4 anos de idade.

\section{Situação e materiais}

Foi semelhante ao Experimento 2, sendo que as sessões experimentais ocorreram no Laboratório de Análise Experimental do Comportamento - LAEC, da Universidade Católica de Goiás. Foi realizada em uma sala experimental de $2 \times 2$ metros, com temperatura e iluminação artificial, contendo um microcomputador com tela sensível ao toque. Ademais seguiu como no Experimento 2.

\section{Procedimento}

Foi semelhante ao Experimento 2, pois nesse Experimento 3 manteve a substituição das figuras do pato e do coelho (Experimento 1) pelas figuras da bola e da casa, como no Experimento 2. O Experimento 3 diferenciou-se do Experimento 2, devido a modificação da posição dos estímulos de comparação na tela do computador, a cada tentativa.

\section{Resultados}

A estrutura das tabelas abaixo é semelhante às apresentadas no Experimento 1 e 2.

\section{Tabela 7}

Porcentagem de respostas corretas apresentadas na Etapa 1 do Experimento 3

\begin{tabular}{|c|c|c|c|c|c|c|}
\hline \multirow{2}{*}{$\begin{array}{l}\text { Estímulos de } \\
\text { comparação }\end{array}$} & \multicolumn{3}{|c|}{ Tentativas 1 e 3} & \multicolumn{3}{|c|}{ Tentativas 2 e 4} \\
\hline & M & $\mathrm{N}$ & $\mathrm{C}$ & M & C1 & C2 \\
\hline Resultados & 0 & 8 & 0 & 5 & 1 & 2 \\
\hline$\%$ & 0 & 100 & 0 & 62,5 & 12,5 & 25,0 \\
\hline
\end{tabular}

Observa-se na Tabela 7 que quando uma palavra nova foi ditada, obteve-se nas tentativas 1 e 3 oito respostas para a figura nova (100\%). Nas tentativas 2 e 4, obteve-se cinco resposta para a máscara $(62,5 \%)$, duas respostas para a figura da bola $(25 \%)$ e uma resposta para a figura do cachorro (12,5\%).

Na Tabela 8, observa-se que quando uma palavra nova acompanhada de "s" foi ditada as resposta ficaram distribuídas entre figura nova e figura com três exemplos novos. Nas tentativas 1 e 3, obteve-se quatro respostas para a figura com três exemplos novos (50\%) e quatro respostas para a figura nova (50\%). Nas tentativas 2 e 4, obteve-se oito respostas para a figura nova $(100 \%)$.

\section{Tabela 8}

Porcentagem de respostas corretas apresentadas na Etapa 2 do Experimento 3

\begin{tabular}{|c|c|c|c|c|c|c|}
\hline \multirow{2}{*}{$\begin{array}{l}\text { Estímulos de } \\
\text { comparação }\end{array}$} & \multicolumn{3}{|c|}{ Tentativas 1 e 3} & \multicolumn{3}{|c|}{ Tentativas 2 e 4} \\
\hline & M & $\mathrm{N}$ & $\mathrm{N}+\mathrm{s}$ & M & $\mathrm{N}$ & C \\
\hline Resultados & 0 & 4 & 4 & 0 & 8 & 0 \\
\hline$\%$ & 0 & 50,0 & 50,0 & 0 & 100 & 0 \\
\hline
\end{tabular}

Na Tabela 9 observa-se que quando uma palavra nova acompanhada de "s" foi ditada na presença de quatro estímulos de comparação, as respostas ficaram distribuídas entre figura nova e figura com três exemplos novos. Nas tentativas 1, 3, 4 e 6, obteve-se nove respostas para a figura com três exemplos novos $(56,2 \%)$ 
e sete respostas para a figura nova (43,7\%). Nas tentativas 2 e 5 , obteve-se oito respostas para a figura nova $(100 \%)$.

\section{Tabela 9}

Porcentagem de respostas corretas apresentadas na Etapa 2 do Experimento 3

\begin{tabular}{|c|c|c|c|c|c|c|c|}
\hline \multirow{2}{*}{$\begin{array}{l}\text { Estímulos de } \\
\text { comparação }\end{array}$} & \multicolumn{3}{|c|}{ Tentativas 1,3, 4 e 6} & \multicolumn{4}{|c|}{ Tentativas 2 e 5} \\
\hline & M & $\mathrm{N}$ & $\mathrm{N}+\mathrm{s} C$ & M & $\mathrm{N}$ & C1 & C2 \\
\hline Resultados & 0 & 7 & 9 & 0 & 8 & 0 & 0 \\
\hline$\%$ & 0 & 43, & 56,20 & 0 & 10 & 0 & 0 \\
\hline
\end{tabular}

Ao descrever as Tabelas 7, 8 e 9, pôde-se observar um total de três respostas (Tabela 7) para figuras conhecidas durante as etapas de teste no Experimento 3.

Para verificar se a frequência de respostas observadas foi significativamente diferente da distribuição da frequência de respostas esperadas nos Experimentos 1, 2 e 3, foi realizado o teste Qui-Quadrado.

Observou-se com a realização do teste Qui-Quadrado para todas as etapas de teste dos três experimentos, que o valor obtido de p é menor que 0,001 , isto é, menor que o nível de significância (5\%). Assim, em todos os três experimentos, a hipótese nula (distribuição de respostas semelhantes dentre os estímulos de uma tentativa) foi rejeitada e a hipótese alternativa (preferência pelo estímulo novo) foi aceita. Verifica-se uma diferença significativa na distribuição das respostas observadas entre os estímulos apresentados, ou seja, estímulos que compartilhavam de uma propriedade "nova" exerceram controle sobre o responder dos participantes nos diferentes experimentos (1, 2 e 3$)$.

\section{Discussão}

Verificou-se no Experimento 3 a diminuição na frequência de respostas para as figuras conhecidas na Etapa 1 do teste. Nas Etapas 2 e 3 não ocorreram respostas para figuras conhecidas quando uma palavra nova com terminação "s" foi apresentada. Desta forma, a suposição apresentada de que a posição dos estímulos de comparação no Experimento 2 estava controlando o responder pode ser considerada verdadeira de acordo com os dados obtidos no Experimento 3, em que nenhuma resposta foi emitida para as figuras conhecidas nas Etapas 2 e 3 de teste em que a dica lexical estava presente.

Observou-se que o comportamento de selecionar figuras novas e figuras com três exemplos novos ocorreu a cada tentativa de teste, apresentando preferência tanto por uma figura nova como por uma figura com três exemplos novos quando um estímulo modelo acompanhado de "s" foi ditado. Com isso, pôde-se verificar o comportamento frequente de selecionar um estímulo novo. $O$ controle que a propriedade "nova" exerceu sobre o responder ficou evidente quando nas Etapas 2 e 3 as respostas dos participantes ficaram distribuídas entre figura nova e figura com três exemplos novos, no Experimento 3.

\section{Discussão Geral}

Com os dados obtidos nos Experimentos 1, 2 e, especificamente, no Experimento 3 , pode-se dizer que a propriedade "nova" exerceu controle sobre a resposta do participante mesmo quando a dica estava presente, lembrando que a dica lexical também era para o participante um estímulo novo e, mais ainda, ela foi acrescida a um estímulo também novo. Isso significa que, provavelmente, ao se fazer um experimento com palavras novas, palavras novas acompanhadas de "s" e palavras conhecidas, as respostas serão emitidas para as palavras novas, independentemente se elas 
têm ou não tem a terminação "s". Observase a distribuição das respostas nas Tabelas 8 e 9, especificamente, nas tentativas em que a palavra nova com terminação "s" foi ditada na presença de figura nova, máscara e figura conhecida. Pode-se calcular que o índice de respostas para figura nova foi de 100\%. E quando a palavra nova com terminação "s" foi ditada na presença de figuras novas e figura com três exemplos novos, as respostas ficaram distribuídas entre essas duas figuras, não ocorrendo preferência por uma ou outra, pois ambas eram novas. Tais dados fortalecem a posição de que palavras novas e não terminações lexicais (plurais, tempos dos verbos, etc) é que controlam a resposta. Tal posição contrasta com a possibilidade levantada por Costa e de Souza (2003). Nunca é demais lembrar que as comparações feitas são entre modelos visuais como "visão de figuras" e modelos auditivos, como "sons vocais" que são comparados entre si pelo participante. Isso remete para os dados dos experimentos de equivalência de estímulos (Saunders, Drake \& Spradlin, 1999; Hubner, 2006; Domeniconi, Costa, de Souza \& de Rose, 2007; De Rose, de Souza, Rossito \& de Rose, 1989) em que desempenhos em discriminações condicionais são estabelecidos por exclusão. Vale relembrar que o presente conjunto de experimentos (Experimento 1, 2 e 3) é sobre controle por estímulos e que há múltiplas formas de controle das respostas feito pelos estímulos controladores (Spradlin, 2003; Dixon, Dixon \& Spradlin, 1983; Borges, 2005).

Um experimento crucial que demonstraria tal controle seria aquele que usasse este tipo de procedimento com "palavras conhecidas" e apenas uma palavra nova. Após a palavra nova se tornar conhecida e passar a fazer parte do vocabulário da criança, essa palavra nova passaria a ser usada no procedimento como palavra conhecida. Ai, então, seria introduzida a "dica", por exemplo, "conjunto de figuras" mais o acréscimo da terminação "s" para a palavra nova. Dessa forma a comparação a ser feita pelo participante relativo a controle por estímulos seria pela propriedade que controla a resposta por exclusão, no caso, a novidade. É bom relembrar que essa é apenas uma das formas possíveis de controle da resposta a ser aprendida, conforme apontado por Spradlin (2003). É ainda possível que em situações de aprendizagem do que é "novo", haja combinação de propriedades dos estímulos para o controle das respostas, como tem sido demonstrado na literatura sobre controle por estímulos (Terrace, 1966/1975).

\section{REFERÊNCIAS}

Baum, W. M. (2006). Compreender o Behaviorismo: Comportamento, cultura e evolução. $2^{\mathrm{a}} \mathrm{ed}$. Porto Alegre: Artmed. Texto original publicado em 2005.

Carey, S. (1982). Semantic development: The state of the art. In E. Wanner \& L. R. Gleitman (Eds.), Language acquisition: the state of the art (pp. 265-293). Cambridge, MA: MIT Press.

Costa, A. R. A., McIlvane, J. W., Wilkinson, K. M., \& de Souza, D. G. (2001). Emergent word-object mapping by children: further studies using the blank comparison technique. The Psychological Record, 51, 343-355.

Costa, A. R. A., \& de Souza, D. G. (2003). Exclusão e formação de classe de estímulos após ensino de relações condicionais independentes e interrelacionadas com crianças com desenvolvimento típico e adultos com deficiência mental. Tese de doutorado. Universidade Federal de São Carlos, São Carlos, SP.

Costa, A. R. A., de Rose, J. C., \& de Souza, D. G. (submetido). Dicas contextuais no aprendizado de vocabulário por crianças pequenas.

de Rose, J. C., de Souza, D. G., Rossito, A. L., \& de Rose, T. M. S. (1989). Aquisição de leitura 
após história de fracasso escolar: equivalência de estímulos e generalização. Psicologia: Teoria e Pesquisa, 5, 325-346.

de Rose, J. C., de Souza, D. G., \& Hanna, E. S. (1996). Teaching reading and spelling: Exclusion and stimulus equivalence. Journal of Applied Behavior Analysis, 29, 451-469.

de Rose, J. C., de Souza, D. G., Rossito, A. L., \& de Rose, T. M. S. (1992). Stimulus Equivalence and generalization in reading after matching to sample by exclusion. In S. C. Hayes \& L. J. Hayes (eds.), Understanding verbal relations (pp. 69-82). Reno, NV: Context Press.

Dixon, L. S. (1977). The nature of control by spoken words over visual stimulus selection. Journal of Experimental Analysis of Behavior, 27, 433-442.

Dixon, M. H., Dixon, L. S., \& Spradlin, J. E. (1983). Analysis of individual differences of stimulus control among developmentally disabled children. Em K. D. Gadow e I. Bialer (Orgs.), Advances in learning and behavioral disabilities (pp. 85-110). New York: JAI Press.

Domeniconi, C., Costa, A. R. A., de Souza, D. G. \& de Rose, J. C. (2007). Responder por exclusão em crianças de 2 a 3 anos em uma situação de brincadeira. Psicologia Reflexão e Critica, 20, 342-350.

Golinkoff, R. M., Mervis, C. B., \& Hirsh-Pasek, K. (1994). Early object labels: the case for a developmental lexical principles framework. Journal of child language, 21, 125-155.

Terrace, H. S. (1975). Control del estímulo. Em W. K. Honing (Org.), Conducta Operante:investigación y aplicaciones (pp. 330- 413). México: Editorial Trillas.

Hubner, M. M. C. (2006). Controle de estímulos e relações de equivalência. Revista Brasileira de Terapia comportamental e Cognitiva, 8, 95-102.

Hutchinson, J. (1986). Chidren's sensitivity to the contrastive use of object category terms. Papers and Reports on Child Language Development, 25, 49-56.

Keller, F. S. \& Schoenfeld, W. N. (1950/1973). Princípios de psicologia. São Paulo: Editora pedagógica e Universidade Ltda e editora da Universidade de São Paulo - EDUSP.
Martins, W. (2008). Software "Aquisição de Vocabulário Infantil versão 1.0".

McIlvane, W. J., Bass, R. W., O’Brien, J. M., Gerovac, B. J., \& Stoddard, L. T. (1984). Spoken and signed naming of foods after receptive exclusion training in severe retardation. Applied Research in Mental Retardation, 5, 1-27.

McIlvane, W. J., Kledaras, J. B., Lowry, M. W., \& Stoddard, L. T. (1992). Studies of exclusion in individuals with severe mental retardation. Research in Developmental Disabilities, 13, 509-532. McIlvane, W. J., Kledaras, J. B., Munson, L. C., King, K. A., de Rose, J. C., \& Stoddard, L. T

(1987). Controlling relations in conditional discrimination and matching by exclusion. Journal of the Experimental Analysis of Behavior, 48, 187-208.

McIlvane, W. J., Munson, L. C., \& Stoddard, L. T. (1988). Some observations on control by spoken words in children's conditional discrimination and matching by exclusion. Journal of Experimental Child Psychology, 45, 472-495.

McIlvane, W. J., \& Stoddard, L. T. (1981). Acquisition of matching-to-sample performances in severe mental retardation: Learning by exclusion. Journal of Mental Deficiency Research, 25, 33-48.

McIlvane, W. J., Wilkinson, K. M., \& de Souza D. G. (2000). As origens da exclusão. Temas em Psicologia, 8, 195-203.

Mervis, C., \& Bertrand, J. (1994). Acquisition of the novel name-nameless category principle. Child Development, 65, 1646-1663.

Moreira, M. B. (2005). Discriminação Simples Simultânea e Responder Relacional. Dissertação de mestrado, Universidade Católica de Goiás, Goiânia, GO.

Saunders, R. R., Drake, K. M. \& Spradlin, J. E. (1999). Equivalence Class Establishment, Expansion and Modification in Preschool Children. Journal of the Experimental Analysis of Behavior, 71, 195-214.

Skinner, B. F (2006). Sobre o Behaviorismo (Trad. Maria da Penha Villalobos). São Paulo: Cultrix. Texto original publicado em 1974.

Spradlin, J. E. (2003). Alternative theories of the 
origin of derived stimulus relations. The Analysis of Behavior, 27, 433-442.

Stromer, R. (1989). Symmetry of control by exclusion in humans' arbitrary matching-to- sample. Psychological Reports, 64, 915-922.

Wilkinson, K. M., \& McIlvane, W. J. (1997). Blank comparison analysis of emergent symbolic mapping by young children. Journal of Experimental Child Psychology, 67, 115- 130.

Vincent-Smith, L., Bricker, D., \& Bricker, W. (1974). Acquisition of receptive vocabulary in the toddler-age child. Child Development, 45, 189-193.

Wilkinson, K. M., Dube, W. V., \& McIlvane, W. J. (1996). A crossdisciplinary perspective on studies of rapid word mapping in psycholinguistics and behavior analysis. Developmental Review, 16, 125148.

Wilkinson, K. M., Dube, W. V., \& McIlvane, W. J. (1998). Fast mapping and exclusion (emergent matching) in developmental language, behavior analysis, and animal cognition research. The Psychological Record, 48, 407 - 423.

Wilkinson, K. M., \& McIlvane, W. J. (1997). Blank comparison analysis of emergent symbolic mapping by young children. Journal of Experimental Child Psychology, 67, 115-130.

Artigo submetido em 6 de julho de 2009 Aceito em 7 de novembro de 2009

Publicação referente ao $2^{\circ}$. Semestre de 2008, impressa em maio de 2011 